\title{
Molecular Dynamics Modeling of Microstructure Evolution During Growth of Amorphous Carbon Films
}

\author{
J. N. Glosli \\ M. R. Philpott \\ J. Belak
}

PEGERTED

FEB 201990

$0 . \$ 1$

This paper was prepared for submittal to the Workshop on Modeling of Industrial Materials

Santa Barbara, California

January 7-11, 1996

January 8, 1996

This is a preprint of a paper intended for publication in a journal or proceedings. Since changes may be made before publication, this preprint is made available with the understanding that it will not be cited or reproduced without the permission of the author. 
This document was prepared as an account of work sponsored by an agency of the United States Government. Neither the United States Government nor the University of California nor any of their employees, makes any warranty, express or implied, or assumes any legal liability or responsibility for the accuracy, completeness, or usefulness of any information, apparatus, product, or process disclosed, or represents that its use would not infringe privately owned rights. Reference herein to any specific commercial product, process, or service by trade name, trademark, manufacturer, or otherwise, does not necessarily constitute or imply its endorsement, recommendation, or favoring by the United States =--.-Government or the University of California. The views and opinions of authors expressed herein do not necessarily state or reflect those of the United States Government or the University of California, and shall not be used for advertising or product endorsement purposes. 


\title{
MOLECULAR DYNAMICS MODELING OF MICROSTRUCTURE EVOLUTION -
} DURING GROWTH OF AMORPHOUS CARBON FILMS

\author{
J.N. Glosli, M.R. Philpott* a ād J. Belak \\ University of California, Lawrence Livermore National Laboratory, \\ P.O. Box 808, Livermore, CA 94550 \\ *IBM Research Division, Almaden Research Center, \\ 650 Harry Road, San Jose, CA 95120-6099
}

\section{Introduction}

Amorphous carbon films approximately $20 \mathrm{~nm}$ thick are used throughout the computer industry as protective coatings on magnetic storage disks $(1,2)$. As storage densities increase, the role of the overcoat becomes increasingly important because of smaller spacings between the recording head and the spinning disk. Furthermore, future generation disks call for an overcoat thickness of $5 \mathrm{~nm}$ or less. These small length scales and the high speed of the spinning disk $(10-30 \mathrm{~m} / \mathrm{s})$ suggest that a molecular dynamics (MD) model (3) might provide useful insight into friction and wear mechanisms when the head and disk make contact.

One of the necessary inputs required to carry out such an MD model is a specification of the position of all of the atoms in the simulation, i.e. a detailed specification of the material microstructure. Such a detailed understanding of the microstructure of amorphous carbon overcoats does not presently exist. Neutron (4) and electron (5) diffraction studies demonstrate that the material is amorphous. Previous classical MD simulations (6-8) yield pair distribution functions in qualitative agreement with the diffraction studies, but they all differ in detail. More recent, quantum mechanical tight-binding MD (TBMD) studies (9-11) give a better description of the interatomic interactions and the chemical hybridization ( $\mathrm{sp}^{2}$-graphite-like versus $\mathrm{sp}^{3}$-diamondlike). However, these studies are presently limited to rather small system sizes and rapid quench rates.

In this paper we present molecular dynamics simulations of the growth of amorphous carbon films deposited onto a diamond substrate using a bond-order potential model (12-14). This classical potential mimics the quantum mechanics allowing carbon to form strong chemical bonds with a variety of hybridizations. It was found that the system formed unphysical bonding configurations without an added torsional energy between $\mathrm{sp}^{2}$ hybridized carbon atoms (14). This torsional energy was included for all results presented here.

\section{Methods}

The simulation starts with a diamond (100) surface (12 layers) held at room temperature (300K) with a Nose-Hoover thermostat (15). The Brenner bond-order potential predicts a $2 \times 1$ reconstruction of the (100) surface. Periodic boundary conditions are applied within the surface and the bottom-most layer of diamond atoms are held rigid. The upper boundary condition is open. Atoms crossing a boundary $10 \mathrm{~nm}$ above the growing surface are removed from the simulation. Surface simulation cell lengths of $2 \mathrm{~nm}$ and $4 \mathrm{~nm}$ are considered and the simulations were run for $10^{7}$ steps with a $1 \mathrm{fs}$ timestep for pure carbon and a $0.5 \mathrm{fs}$ timestep for mixed carbon and hydrogen films. Carbon and hydrogen atoms are rained down onto random surface positions at a rate of $1 \mathrm{atom} / \mathrm{ps}$ with average kinetic energies ranging from $1 \mathrm{eV}$ to $100 \mathrm{eV}$. Significant heat is generated when the incident atoms form chemical bonds with the surface. In addition to coupling the diamond sub- 

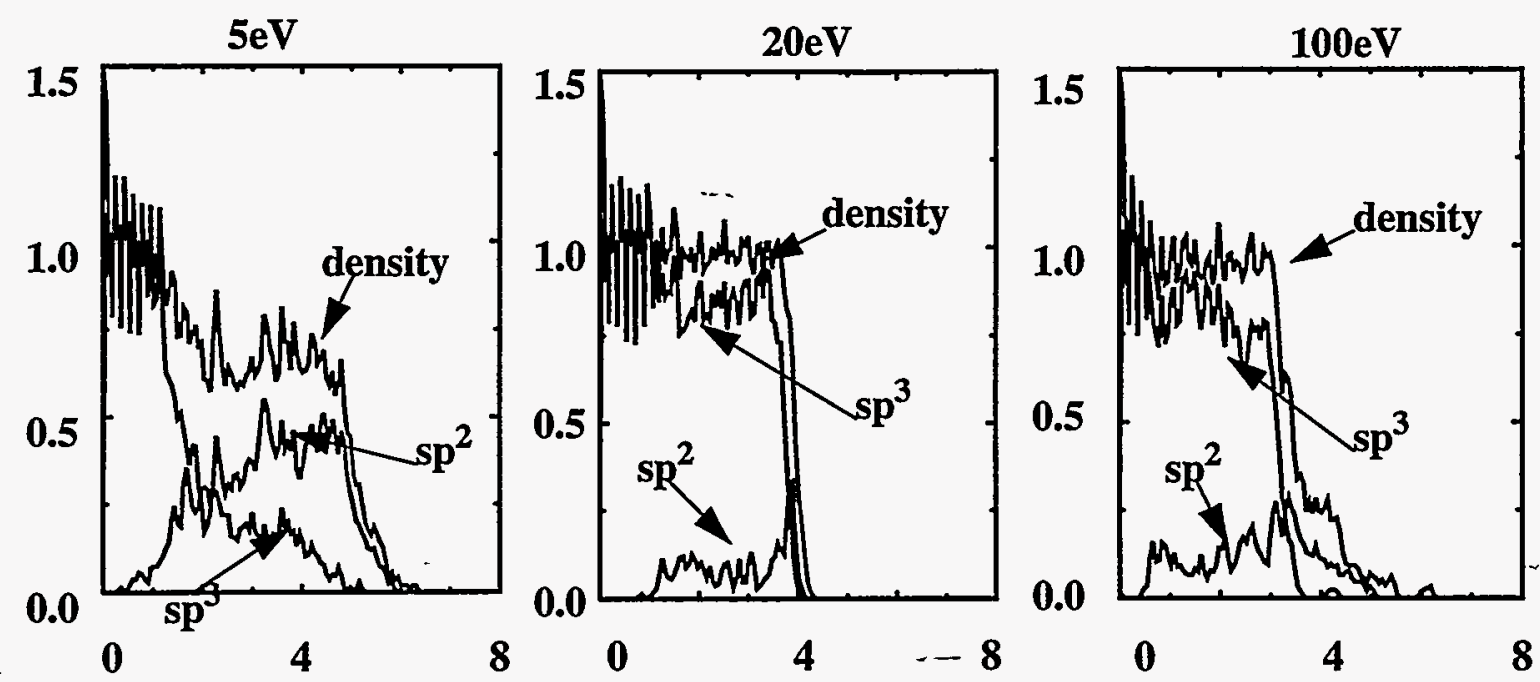

Figure 1. The growth of amorphous carbon films (a:C) on diamond (100) at deposition ener-. gies $5 \mathrm{eV}, 20 \mathrm{eV}$, and $100 \mathrm{eV}$. Density profiles are normalized by diamond density $(3.5 \mathrm{gm} / \mathrm{cc})$ and are shown as a function of height through the film (in $\mathrm{nm}$ ).

strate to a thermostat we have explored using a thermostat boundary that tracks the growing surface and a hybrid method in which only the diamond substrate is held at room temperature during the first half of the time interval between deposited atoms and the entire system is quenched to room temperature during the latter half. This latter method effectively emulates a much slower deposition rate.

\section{Results and Discussion}

The resulting density profiles as a function of height through the film for three growth simulations $(5 \mathrm{eV}, 20 \mathrm{eV}$, and $100 \mathrm{eV})$ of amorphous carbon (a:C) are shown in Figure 1 after several nanoseconds of simulation time (16). The microstructure of the films and the morphology of the growth process varies significantly with the deposition energy. At low energy, the film is at lower density $\left(\rho / \rho_{D} \sim 0.7\right)$ with a significant fraction $(70 \%)$ of $\mathrm{sp}^{2}$ hybridized carbon atoms. There are large open regions in the film and chain-like $\left(\mathrm{sp}^{1}\right)$ structures growing on the surface. Increasing the energy to $20 \mathrm{eV}$, we find a very compact film at near diamond density $\left(\rho / \rho_{\mathrm{D}} \sim 1\right)$ with only $10 \% \mathrm{sp}^{2}$ carbons in the film. The interface between the film and the vapor is very smooth. This interface becomes very diffuse at high energy $(100 \mathrm{eV})$ where many carbon atoms leave through the vapor. The resulting film is less dense $\left(\rho / \rho_{D} 0.95\right)$ though not containing the open regions found at low energy. The $\mathrm{sp}^{2}$ fraction has increased to about $20 \%$.

The density profiles for films grown with hydrogen are shown in Figure 2. Here we deposit an-equal concentration of carbon and hydrogen atoms, though only $20-30 \%$ of the atoms in the films are hydrogens. The rest of the hydrogen atoms leave through the vapor as small hydrocarbon molecules. The presence of hydrogen lowers the number of $\mathrm{sp}^{2}$ carbons at low energy and increases the number at high energy. The most pronounced effect of the hydrogen, however, is to lower the stress in the films. At $20 \mathrm{eV}$ the stress in the hydrogenated film is 4 times smaller than for the pure carbon film at the same deposition energy. The calculated value of compressive stress for our $20 \mathrm{eV}$ film (13GPa) is in qualitative agreement with experiment (8GPa) though we note that the small size of our simulation cell inhibits large scale relaxation mechanisms. Large surface cells need to be simulated to further quantify the results. 

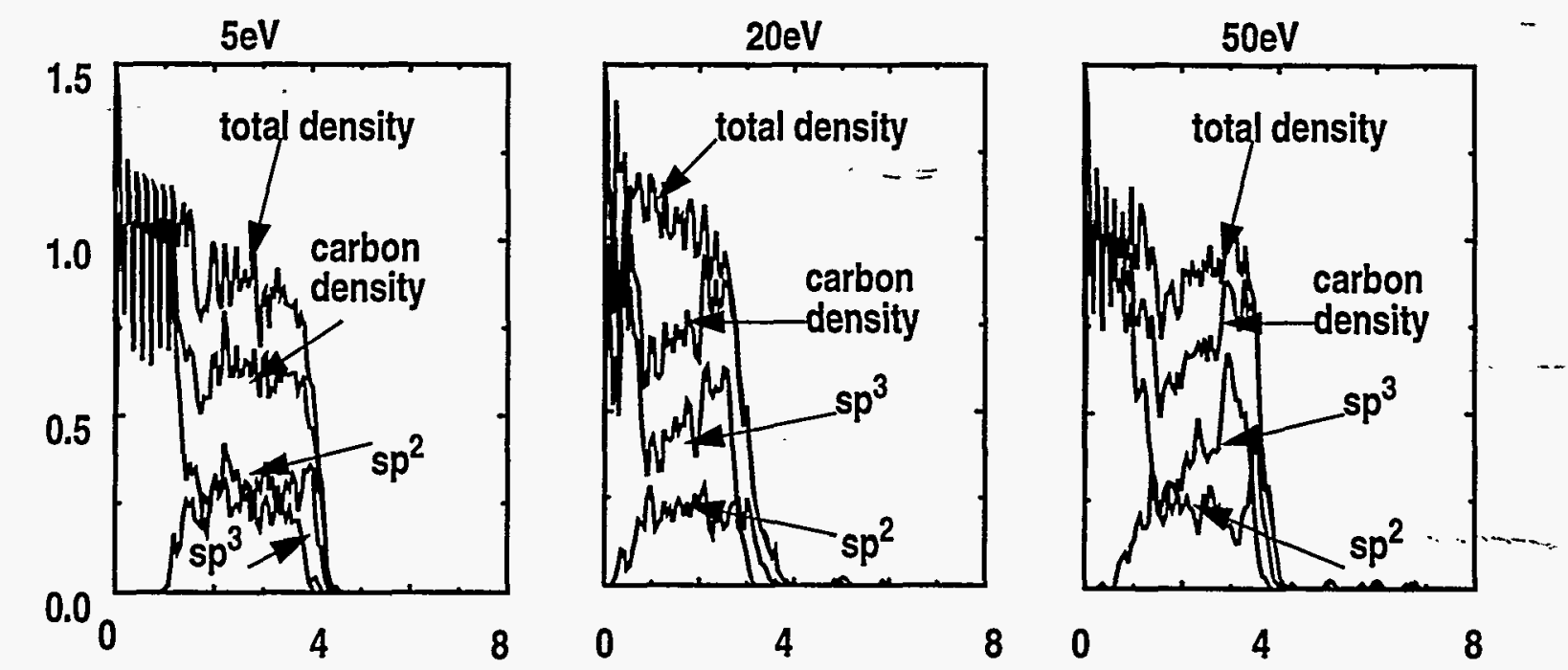

Figure 2. The density profiles in simulated amorphous hydrogenated carbon films (a:CH) at deposition energies of $5 \mathrm{eV}, 20 \mathrm{eV}$, and $50 \mathrm{eV}$ as a function of position in the film in $\mathrm{nm}$. The.4 curves are the total density, the density of all forms of carbon, the density of $\mathrm{sp}^{2}$ carbon and the density of $\mathrm{sp}^{3}$ carbon.

The loading curve (force on the tool as a function of tool height) is shown in Figure 3 for our $20 \mathrm{eV}$ a:C film(17). The indenter tip is cleaved from a diamond lattice and blunted to create a radius of about $1 \mathrm{~nm}$. The tip atoms are held rigid during indentation and interact with the surface atoms through a truncated Lennard-Jones potential. Indentation was performed at $35 \mathrm{~m} / \mathrm{s}$. This rate is comparable to the sliding speeds at the head-disk interface in magnetic recording disks. The steps in the curve indicate the onset of plasticity. The curve is reversible before the first step while a hysteresis loop is seen beyond the first step. This step-like structure is probably characteristic of covalently bonded materials and represents plastic flow through a rapid rearrangement of the bonding network. The lengthscale of our simulations is too small to observe the formation of cracks. The hardness calculated from this simulation is $75+/-25 \mathrm{GPa}$, with the error arising from our estimate of the contact area. This value is about twice the measured hardness on dense amorphous carboñ films $(1,2,18)$.

\section{Summary}

Classical molecular dynamics simulations using Brenner's bond-order potential model with additional torsional energy terms give quantitative agreement with tight-binding molecular dynamics for the microstructure of quenched amorphous carbon films. For a 64 atom quench we find $56 \mathrm{sp}^{3}$ and $8 \mathrm{sp}^{2}$ carbons, 6 of which come in pairs. The recent TBMD of Drabold, et al.(11) found $6 \mathrm{sp}^{2}$ carbons, all of which were paired. It appears the potential gives a reasonable description of the energy surface for carbon covalent bonding, though we do not know to what extend the kinetics (or transition states) are correct.

The deposition simulations show some interesting features. Hydrogen increases the $\mathrm{sp}^{3}$ bonding of films grown at low energy and plays an important role in releasing residual stress in the films. The stress in our simulated films reaches a maximum of about $18 \mathrm{GPa}$ for pure carbon at a deposition energy of 50eV. Experiments (5) for deposition of very high carbon concentration show a maximum stress of about $8 \mathrm{GPa}$ at a deposition energy of $20 \mathrm{eV}$. The discrepancy is most likely due to the pure carbon in our simulation (no impurities) and the small size of our simulation cell. Both of which will lower the stress. The density is maximal near the deposition energies (20- 


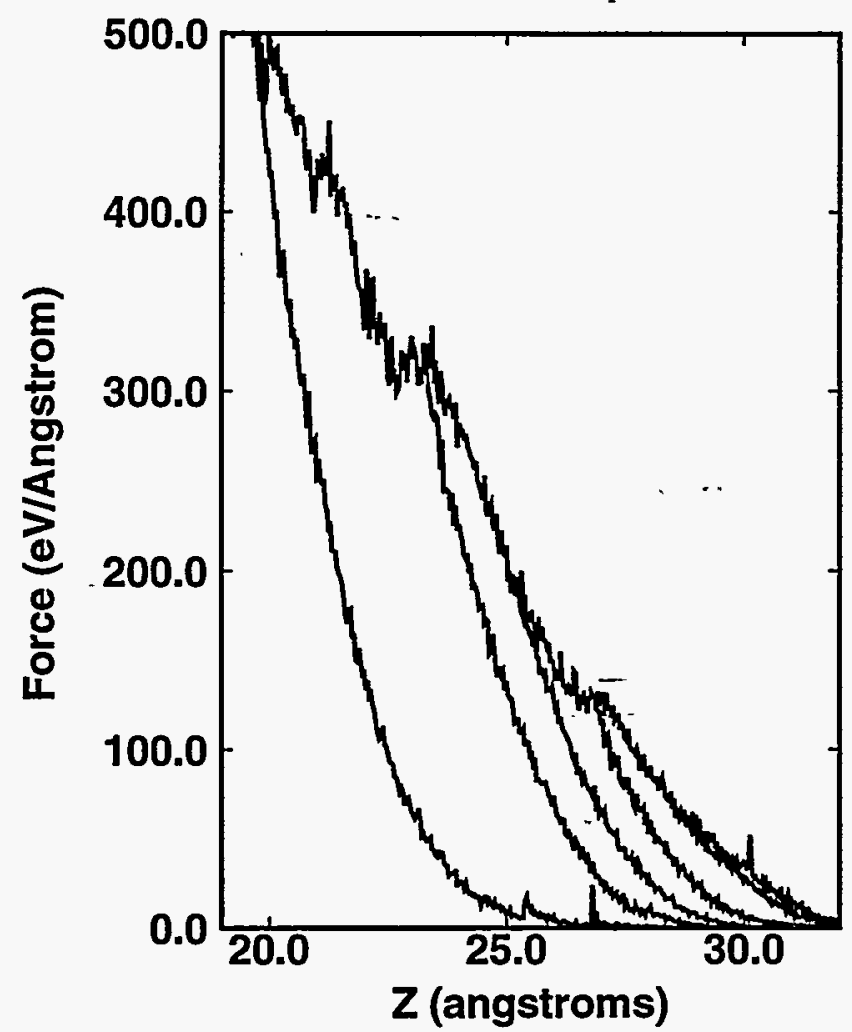

Figure 3. The indentation loading curve for the $20 \mathrm{eV}$ simulation shown in Figure 1. The steps occur during plastic yielding of the surface. Reversing the tip after these yielding events leads to the hysteresis as shown.

$40 \mathrm{eV}$ ) used to grow films on magnetic disks. We might expect these films with high density and lower stress to have good tribological properties. Another interesting aspect of the simulations is the thickness of the interface between the growing film and the vapor above. This thickness is large (several nanometers) at both low and high deposition energy and minimal at moderate deposition energy $(20 \mathrm{eV})$. With the trend towards thinner overcoats on magnetic disks $(5 \mathrm{~nm})$, the properties of the overcoat may be dominated by interfacial phenomena.

The hardness calculated for the densest simulated films is somewhat larger than experiment by about a factor of two. This is somewhat surprising in that we might not expect a lengthscale dependence for an amorphous film (prior to the formation of cracks). The difference may be due to impurities (always present in experiment but absent from the simulation) or might suggest the need for further improvement of the bond-order potential model.

\section{Acknowledgments}

Work performed under the auspices of the U.S. Department of Energy by the Lawrence Livermore National Laboratory under contract No. W-7405-ENG-48.

\section{References}

1. H-C Tsai and D.B. Bogy, J. Vac. Sci. Technol. A 5, 3287 (1987).

2. A. Gill, Wear 168, 143 (1993).

3. M.P. Allen and D.J. Tildesley, Compüter Simulation of Liquids (Oxford, Clarendon, 1987). 4. F. Li and J.S. Lannin, Phys. Rev. Lett. 65, 1905 (1990). 
5. D.R. McKenzie, D. Muller, and B.A. Pailthorpe, Phys. Rev. Lett. 67, 773 (1991).

6. J. Tersoff, Phys. Rev. Lett. 61, 2879 (1988).

7. B.A. Pailthorpe, J. Appl. Phys. 70, 543 (1991).

8. H.-P. Kaukonen and R.M. Nieminen, Phys. Rev. Lett. 68, 620 (1992).

9. C.Z. Wang, K.M. Ho, and C.T. Chan, Phys. Rev. Lett. 70, 611 (1993).

10 C.Z. Wang and K.M. Ho, Phys. Rev. Lett, 71, 1184 (1993).

11. D.A. Drabold, P.A. Fedders, and P. Stumm, Phys. Rev. B 49, 16415 (1994).

12. J. Tersoff, Phys. Rev. Lett. 61, 2879 (1988).

13. D.W. Brenner, Phys. Rev. B 42, 9458 (1990).

14. D.W. Brenner, J.A. Harrison, C.T. White, and R.J. Colton, Thin Solid Films 206, 220 (1991).

15. W.G. Hoover, Phys. Rev. A 31, 1695 (1985).

16. J.N. Glosli, J. Belak, and M.R. Philpott: 'Ultra-Thin Carbon Coatings for Head-Disk Interface Tribology,' in Thin Films: Stresses and Mechanical Properties V, S.P. Baker, C.A. Ross, P.H. Townsend, C.A. Volkert, and P. Borgesen eds., Materials Research Society Symposium Proceedings 356, MRS, Pittsburgh, USA, 1995.52-

17. J.N. Glosli, M.R. Philpott, and J. Belak: 'Molecular Dynamics Simulation of Mechanical Deformation of Ultra-Thin Amorphous Carbon Films,' in Mechanical Behavior of Diamond and Other Forms of Carbon, M.D. Drory, M.S. Donley, D. Bogy, and J.E. Field eds., Materials Research Society Symposium Proceedings, MRS, Pittsburgh, USA, 1995.

18. J.B. Pethica, P. Koidl, J. Gobrecht, and C. Schuler, J. Vac. Sci. and Tech. A, 3, 2391, (1985). 

* 
Tcchnical Information Department - Lawrence Livermore National Laboratory University of California - Livermore, California 94551

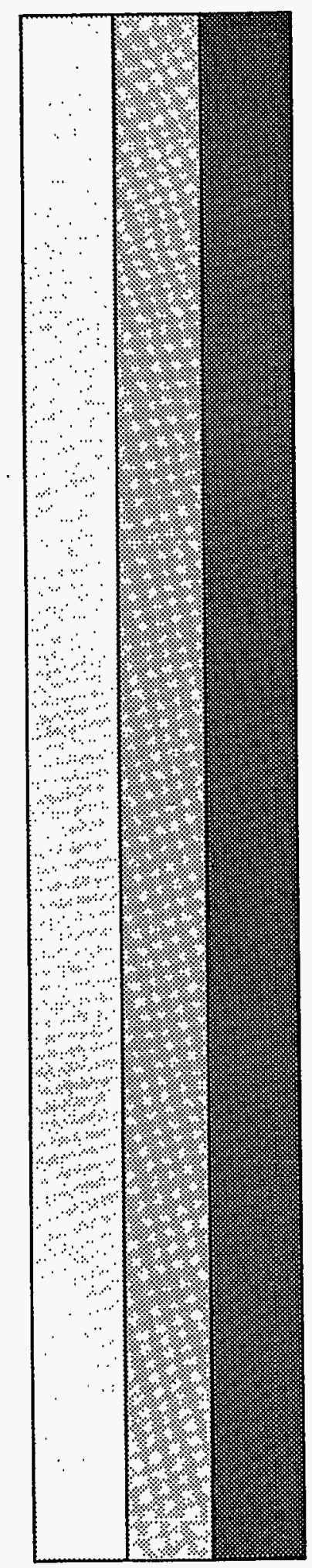

